\title{
Dissociative CdSe/ZnS Quantum Dot-Molecule Complex for Luminescent Sensing of Metal lons in Aqueous Solutions
}

\author{
A. V. Baranov \\ State University of Informational Technologies,Mechanics and Optics, St. Petersburgh
}

A. O. Orlova

State University of Informational Technologies, Mechanics and Optics, St. Petersburgh

V. G. Maslov

State University of Informational Technologies, Mechanics and Optics, St. Petersburgh

See next page for additional authors

Follow this and additional works at: https://arrow.tudublin.ie/engscheceart

Part of the Electrical and Computer Engineering Commons

\section{Recommended Citation}

Baranov, A.V. et al:Dissociative CdSe/ZnS quantum dot-molecule complex for luminescent sensing of metal ions in aqueous solutions. J. Appl. Phys. 108, 074306 (2010); http://dx.doi.org/10.1063/1.3490218

This Article is brought to you for free and open access by the School of Electrical and Electronic Engineering at ARROW@TU Dublin. It has been accepted for inclusion in Articles by an authorized administrator of ARROW@TU Dublin. For more information, please contact arrow.admin@tudublin.ie, aisling.coyne@tudublin.ie, gerard.connolly@tudublin.ie.

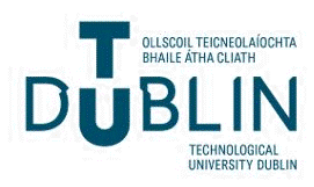




\section{Authors}

A. V. Baranov, A. O. Orlova, V. G. Maslov, Yu. A. Toporova, E. V. Ushakova, A. Federov, M. V. Artemyev, T. S. Perova, and Kevin Berwick 


\title{
DISSOCIATIVE CdSe/ZnS QUANTUM DOT-MOLECULE COMPLEX FOR
}

\section{LUMINESCENT SENSING OF METAL IONS IN AQUEOUS SOLUTIONS}

A. V. Baranov, ${ }^{1, a)}$ A. O. Orlova, ${ }^{1}$ V. G. Maslov, ${ }^{1}$ Yu. A. Toporova, ${ }^{1}$ E. V. Ushakova, ${ }^{1}$ A. V. Fedorov, ${ }^{1}$ M. V. Artemyev, ${ }^{2}$ T. S. Perova ${ }^{3}$ and K. Berwick ${ }^{4}$

${ }^{1}$ Center of Information Optical Technologies, State University of Informational Technologies, Mechanics and Optics, 197101 St-Petersburg, Russia

${ }^{2}$ Institute for Physico-Chemical Problems, Belarussian State University, 220080 Minsk, Belarus ${ }^{4}$ Department of Electronic and Electrical Engineering, Trinity College, University of Dublin, Dublin 2, Ireland

${ }^{3}$ Department of Electronic and Communications Engineering, Dublin Institute of Technology, Dublin 8, Ireland

a) Electronic mail: a_v_baranov@yahoo.com

\begin{abstract}
The optical properties of dissociative luminescent sensors based on a complex consisting of highly luminescent hydrophobic core/shell CdSe/ZnS quantum dots and 1-(2-pyridilazo)-2naphtol (PAN) molecules in organic solutions and a polymer film are reported. It is demonstrated, using $\mathrm{Ni}^{2+}$ and $\mathrm{Co}^{2+}$ ions as an illustratoiveillustrative example, that the QD/PAN sensor may have applications in the quantitative luminescent sensing of metal ions in aqueous solutions.
\end{abstract}




\section{Introduction}

Luminescent sensors based on complexes consisting of a semiconductor nanocrystal (quantum dot, QD) with organic dye molecules bound to its surface are highly sensitive detectors of various species, including metal ions, and have applications in biology, medicine and ecology [1]. Using a QD as a sensor luminophor offers considerable advantages over conventional organic and protein based fluorophores [2]. QDs have absorption and photoluminescence excitation bands over a broad spectral range. The molar extinction coefficients of QDs are an order of magnitude larger than those of conventional dyes. QDs also have a narrow and symmetric photoluminescence (PL) band, with quantum yields of up to $80 \%$, together with a high resistance to both photobleaching and chemical degradation. Finally, because of quantumconfinement effects, PL emission of QDs can be tuned across a broad spectral range simply by changing the size of the nanocrystal.

QD luminescence in these QD/molecule sensors is initially quenched by Fluorescence Resonant Energy Transfer (FRET) from the QD to the dye molecule. A large value of the overlapping integral can be obtained by optimizing the QD size. One type of these sensors utilizes the fact that the absorption spectrum of the dye molecule is modified in the presence of an analyte $e_{2}$ resulting in the a decrease of the overlapping integral value and an increase of $\underline{\text { in the }}$ QD PL intensity [3]. A variety of such-these organic dye molecules are used as indicators in quantitative colorimetric analysis of metal ions in water solutions [4]. Alternatively, the presence of the an analyte may result in the dissociation of the QD/molecule complex that causes theand a resultant increase in the QD PL intensity [5]. In either case, the PL intensity is a function of the analyte concentration, and so can be used for analytical purposes.

In this paper, we report the optical properties of dissociative luminescent sensors for $\mathrm{Ni}^{2+}$ and $\mathrm{Co}^{2+}$ ions, based on a complex consisting of highly luminescent hydrophobic core/shell CdSe/ZnS quantum dots and 1-(2-pyridilazo)-2-naphtol (PAN) molecules bound to the QD surface. The azo-dye PAN is a well-known metallochromic indicator for the colorimetric 
determination of a variety of metal ions, including $\mathrm{Ni}^{2+}$ and $\mathrm{Co}^{2+}[6,7 \text { and references therein }]_{\text {. }}$

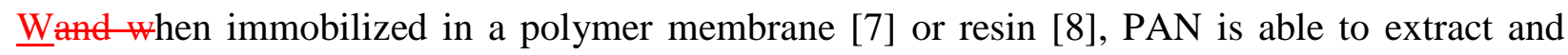
concentrate metal ions from water aqueous solutions, allowing a more sensitive determination of analytes. The advantage of our approach is to that measurement of the changes in the PL response of the QD/PAN sensor induced by the presence of analyte that is at least an order of magnitude more sensitive than colorimetric measurements.

\section{Experimental}

\subsection{Materials and apparatus}

The 1-(2-pyridilazo)-2-naphtol (PAN) and organic solvents used in this study were purchased from Fluka and used as received. All metal salts $\left(\mathrm{NiSO}_{4}, \mathrm{Co}\left(\mathrm{NO}_{3}\right)_{2} 6 \mathrm{H}_{2} \mathrm{O}\right.$, and $\mathrm{Zn}\left(\mathrm{CCl}_{3} \mathrm{COO}\right)_{2}$ $2 \mathrm{H}_{2} \mathrm{O}$ ) were of analytical reagent grade and dissolved in bidistilled water. The two-component polyurethane adhesive Novacote ${ }^{\circledR}$ NC-250-A (resin) + CA-350 (hardener) [9] used for the preparation of the polymer films were purchased from Coim UK, Ltd.

CdSe/ZnS core/shell quantum dots were synthesized according to a protocol adapted from Ref. [10] and described in detail elsewhere [11]. The QDs have a CdSe core the desired diameter and an epitaxially grown ZnS shell approximately two monolayers in thickness. The QDs exhibit strong band-edge PL with a quantum yield (QY) of nearly $50 \%$ in a toluene solution. The quantum dot surface is covered in trioctylphosphine oxide (TOPO) molecules coupled with the shell Zn ions by coordination bonds.

A Shimadzu UV Probe 3600 spectrophotometer and a Varian Fluorescence, Cary Eclipse spectrophotometer were used for recording the absorption and PL spectra. A MicroTime100, PicoQuant Time-resolved Fluorescence microscope was used for the PL decay time measurements. pH values were determined with-using a Hanna Instruments HI98108 pH Meter. 


\subsection{Sample preparation}

QD/PAN complexes in organic solutions (chloroform, carbon tetrachloride, hexane and toluene) were prepared by adding PAN solutions with various concentrations ( $\left.\mathrm{C}_{\mathrm{PAN}}\right)$ to a $\mathrm{QD}$ solution with a concentration $\left(\mathrm{C}_{\mathrm{QD}}\right)$ of about $5 \underline{\times 10^{-7}} \mathrm{M}$ in such a way that the $\mathrm{C}_{\mathrm{QD}}$ : $\mathrm{C}_{\mathrm{PAN}}$ ratio $(\boldsymbol{R})$ varied from 3:1 to 1:10. The $\underline{R}$ reference solutions of PAN chelates with $\mathrm{Zn}^{2+}, \mathrm{Co}^{2+}$, and $\mathrm{Ni}^{2+}$ ions have were been-prepared by adding PAN solution to solutions of metal salts and stirring [12].

The polymeric films with embedded QD/PAN complexes were fabricated as follows. First, toluene solutions of CdSe/ZnS QDs $\left(\mathrm{C}_{\mathrm{QD}} \sim 1.3 \underline{\mathrm{x}} 10^{-4} \mathrm{M}\right)$ and PAN with a $\mathrm{C}_{\mathrm{PAN}}$ in the range of $0.4-13 \underline{\mathrm{x}} 10^{-4} \mathrm{M}$ were prepared. ThenNext, $1.5 \mathrm{ml}$ of the QD solution and $1.5 \mathrm{ml}$ PAN solution were added to $1 \mathrm{ml}$ of Novacote ${ }^{\circledR}$ NC-250-A + CA-350 adhesive with a component ratio of 1:20. The mixture was stirred for 5 minutes and then placed in an ultrasonic bath for 10 minutes. Finally, the mixture was deposited on a 12 micron thickness $\underline{\text { thick, }}$ polyethyleneterephtalate substrate and dried, in accordanceing to with the adhesive specification, at $\mathrm{T} \sim 100{ }^{\circ} \mathrm{C}$ for 15 minutes, followed by a room temperature drying step for $\sim 20$ hours. Thin polymer films of 1-3 micron thickness with embedded QD/PAN complexes were obtained as a result of this procedure. Taking into account the 1.6 times-_solidification shrinkage of the films, the concentration of the components was calculated to be $\mathrm{C}_{\mathrm{QD}}=4_{-}-\underline{\mathrm{x}} 10^{-4} \mathrm{M}$ and $\mathrm{C}_{\mathrm{PAN}} \approx(1.2-$ 40) $\lcm{\times} 10^{-4} \mathrm{M}$, resulting in an so, that the $\boldsymbol{R}$ value that varied from 3:1 to 1:10. We also prepared also the-reference samples of polymeric films, containing either QDs or PAN with the same molar concentrations. Polymeric films containing PAN chelates with $\mathrm{Zn}^{2+}, \mathrm{Co}^{2+}$, and $\mathrm{Ni}^{2+}$ ions were prepared by immersion of the film containing PAN in an the aqueous solution of the corresponding metal salt.

\section{Results and discussion}

3.1. QD/PAN complex formation in organic solutions 
We studied firstOur initial investigations focused on a study of the formation of QD/PAN complexes in chloroform, carbon tetrachloride, hexane and toluene by means ofusing $\underline{\text { PL and }}$ absorption spectroscopyabsorption/PL spectroscopy. In order to carry out reliable absorption/luminescencespectroscopy measurements, diluted solutions of the reagents, with $\mathrm{C}_{\mathrm{QD}}$

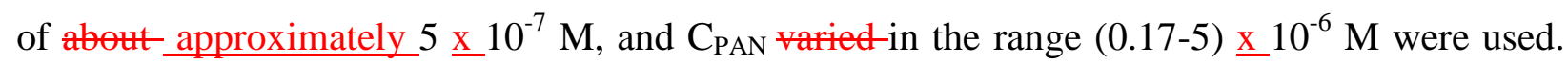
Both hereIn this, and_and_in subsequent experiments, CdSe/ZnS QDs with diameters of $2.5 \mathrm{~nm}$ and $530 \mathrm{~nm}$ PL bands were mainly utilizedused. Figure 1(1,2) shows the absorption and PL spectra of the $2.5 \mathrm{~nm}$ QDs in toluene. Figure 1(3) demonstrates-illustrates the absorption spectra of neutral PAN in a toluene solution at the $\underline{\text { a }}$ concentration of $10^{-6} \mathrm{M}$.

The addition of PAN to the QD solutions results in a dramatic change_sin the absorption spectrum of the mixture. This change is that is illustrated in Fig. 1(4) for a toluene solution. The characteristic absorption spectrum of the PAN/ $\mathrm{Zn}^{2+}$ complexes in toluene with a $560 \mathrm{~nm}$ band [13] appears in the spectrum of the mixture. The appearance of the characteristic PAN/ $\mathrm{Zn}^{2+}$ absorption spectrum points-indicates to the formation of chelate compounds between the PAN molecules and the surface $\mathrm{Zn}$ ions of $\underline{\text { of the }} \mathrm{QD}$ shell, i.e. QD/PAN complexes. It is not surpriseThis is unsurprising, since since-PAN can form a nonluminescent, colored chelate complex with $\mathrm{Zn}^{2+}$ ions [13]. The characteristic time frequired for this process decreases with an increase in the reagent concentrations that are expected for bimolecular reactions. For a $\mathrm{C}_{\mathrm{QD}}=5$ $\underline{\mathrm{x}} 10^{-7} \mathrm{M}$ and $\mathrm{C}_{\mathrm{PAN}}=10^{-6} \mathrm{M}$, the increase in the intensity of the characteristic $560 \mathrm{~nm}$ absorption band saturates in-ca. 3 hours after the addition of the -PAN addition-at room temperature (curve 4 in Figure 1). Such-This long_-characteristic time_-of for QD/PAN formation may be due to the slow substitution of the surface TOPO molecules by PAN.

Simultaneously, a reduction in the QD PL intensity by two orders of magnitude was observed $_{2}$ indicating that practically all of the QDs bind to form nonluminescent QD/PAN

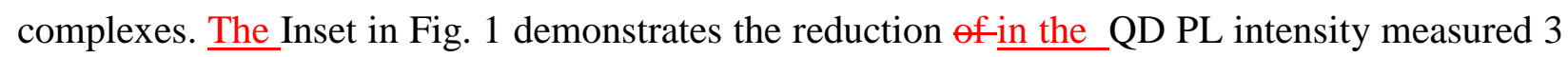
hours after the addition of the PAN PAN addition-for different-various $\boldsymbol{R}$ values. We found that 
the mean PL decay times of about $\underline{\text { around } 9} 9$ ns appeared to be-are practically the sameidentical

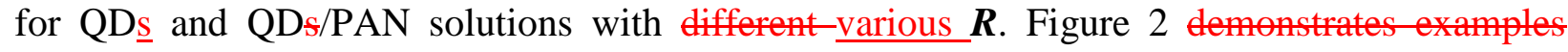
өfillustrates representative PL decay curves $\mathrm{F}(\mathrm{t})$ for pure QDs and for QD/PAN mixtures in toluene. These-curves are well fitted by a sum of 2 exponentials $F(t)=\sum \alpha_{i} \exp \left(-t / \tau_{i}\right)$, where $\alpha_{i}$ and $\tau_{i}$ are the amplitude and decay time of the jth-ith component, respectively. The mMean time for the multiexponential PL decays is $\langle\tau\rangle=\sum \alpha_{i} \tau_{i}^{2} / \alpha_{i} \tau_{i}$, where $\alpha_{i} \tau_{i}$ are the weights of the jth-ith component in the PL emission. The same mean decay time $\langle\tau\rangle$ for solutions of free QDs and the QD/PAN mixture points to the factsuggests that the emission $\theta$ from QDs in the QD/PAN mixture principally belongs originates from mainly to - unbound nanocrystals yet presented in the mixture. HenceTherefore, a-static quenching of the QD PL takes place and the reduction in the PL observed PL intensity reduction-observed with increasing PAN concentration reflects the formation of nonluminescent QD/PAN complexes. Analysis of the Analysis of PL intensity dependence on the-PAN concentration in terms of the theory of static quenching [14] showed that complexes consisting of one QD and one PAN molecule dominate at $\boldsymbol{R}$ values lower than 1:2. We think believe that the QD PL quenching in the QD/PAN complexes is most likely eaused-due to by-FRET from the $2.5 \mathrm{~nm}$ QDs to the PAN/Zn ${ }^{2+}$ compound due to the large value of the overlapping integral between the QD PL band and the absorption band of the PAN/Zn ${ }^{2+}$ compound (Fig. 1). Identifying The FRET as the origin-mechanism responsible for quenching is supported by the fact that no noticeable-significant decrease in PL decrease-was observed in the an experiment with QDs of $5.3 \mathrm{~nm}$ in-diameter mand a PL band located at 630 nm when the spectral overlapping of the PAN $/ \mathrm{Zn}^{2+}$ absorption and the QDs PL bands is negligible.

Similar changes in the absorption and PL spectra of a mixture of QD and PAN solutions have been observed for other hydrophobic solvents, such as chloroform, carbon tetrachloride and hexane, supporting the theory that formation of nonradiating QD/PAN complexes is occurring, where PAN molecules are bound to QD shell Zn ions. 
The QD/PAN complexes prepared demonstrated remarkable PL behavior. When mixing the QD/PAN organic solution with water containing $\mathrm{Co}^{2+}$ or $\mathrm{Ni}^{2+}$ ions, an the increase in the QD PL with ion concentration was observed. This raises the possibility that QD/PAN complexes could be used for luminescence sensing of $\mathrm{Co}^{2+}$ or $\mathrm{Ni}^{2+}$ ions in solutions. However, in practice, the use of a the exploitation of-QD/PAN PL sensor in form of -solution form suffers from somehas obvious limitations-obvious drawbacks, necessitating a redirection of our - Therefore, efforts to an shifted our efforts to the examination of the sensing properties of QD/PAN complexes in polymer films.

\subsection{Dissociative luminescent sensor for $\mathrm{Co}^{2+}, \mathrm{Ni}^{2+}$ and $\mathrm{H}^{+}$ions}

Thin polymer films with thicknesses of 1-3 microns containing QD/PAN complexes have beenwere prepared ${ }_{2}$ as described in the Experimental section. Thin polymer films allow precise absorption and luminescence measurements at QD and PAN concentrations in films of about $10^{-4}$ $\mathrm{M}$, that is, three orders of magnitude higher than those in organic solutions. It is expected als $\Theta$ that polymeric films may work as a-preconcentrators of the detecting ions being sensed, increasing the sensitivity of the sensor.

Preliminary examinations of polymeric films with QD/PAN complexes show exhibit PL behavior similar to QD/PAN in solution: QD PL quenching with increased $\mathrm{C}_{\mathrm{PAN}}$ concentration (Fig. 3a) and the-a mean QD PL decay time independent on-of the PAN molar ratio. In parallel with the decrease in QD PL intensity, a characteristic PAN $/ \mathrm{Zn}^{2+}$ absorption spectrum emerges, totally dominating the film absorption at $\boldsymbol{R}=1: 10$. This is illustrated in Fig. 3b, where a comparison of the absorption spectra of polymeric films containing free QDs (1), QD/PAN complexes $(2,3)$ and PAN/Zn ${ }^{2+}$ chelate compounds (dashed line) are presented. The reference polymeric film with $\mathrm{PAN} / \mathrm{Zn}^{2+}$ chelates was prepared by submerging a film with PAN in an aqueous solution of $10^{-3} \mathrm{M} \mathrm{Zn}\left(\mathrm{CCl}_{3} \mathrm{COO}\right)_{2}$ for 3 hours at room temperature. 
For the experiments on $\mathrm{Co}^{2+}$ sensing, a number of few QD/PAN films with $\boldsymbol{R}$ equal to 1:10 and 1:1 were prepared and submerged in aqueous solutions with a $\mathrm{pH}$ of 6.5 containing $\mathrm{Co}^{2+}$ ions at various $\mathrm{C}_{\mathrm{Co}}$ concentrations, and kept submerged for 5 hours at room temperature. The diffusion of $\mathrm{Co}^{2+}$ ions into the film results in changes in the film absorption spectra and an increase in the QD PL intensity. Figure 4 illustrates changes in the absorption spectra of QD/PAN films with $\boldsymbol{R}$ equal to of $1: 10$ after treatment in cobalt nitrate solutions. This treatment leads to the-a damping of the PAN/ $\mathrm{Zn}^{2+}$ absorption bands (1) and to the appearance of new absorption bands at $585 \mathrm{~nm}$ and $630 \mathrm{~nm}(2,3)$. These bands are characteristic of the PAN/Co ${ }^{2+}$ chelate compound, as can be seen by comparison with the absorption spectrum of a film containing PAN after immersion in the cobalt nitrate solution (4). In figure 4_(Inset), the increase in QD PL intensity with cobalt ion concentration is illustrated. Significantly, a mean luminescence decay time of $\sim 10$ ns was measured from-for these samples. This decay time, as well as the spectral position and linewidth of the PL band, eoincides - is very similar to well with those from CdSe/ZnS quantum dots embedded in the polymer film. This fact-allows us to assign this PL band to the band-edge luminescence of free QDs.

Similar absorption/luminescence changes were observed for polymeric film containing QD/PAN treated in a $6.5 \mathrm{pH}$ aqueous solution of $\mathrm{Ni}^{2+}$ ions (Fig. 5). In this case, the characteristic absorption bands of a PAN/Ni ${ }^{2+}$ chelate at $530 \mathrm{~nm}$ and $570 \mathrm{~nm}$ appear in the film spectrum (curves 2 and 3) with a simultaneous damping reduction in of the PAN/ $\mathrm{Zn}^{2+}$ absorption bands (1). The appearance of these bands was accompanied by an increase in the QD PL signal ${ }_{2}$ with an intensity which is proportional to the $\mathrm{Ni}^{2+}$ ion concentration (Inset in Fig.5). As a result, we conclude that dissociation of QD/PAN complexes occurs in the presence of cobalt or nickel ions, followed by the formation of the stable, nonluminescent chelate compounds $\mathrm{PAN} / \mathrm{Co}^{2+}$ or PAN/Ni ${ }^{2+}$, leaving highly luminescent free QDs. The dissociation of the QD/PAN complexes by Co or Ni ions may be explained by the relatively weak binding of the PAN molecule with-to the QD shell Zn ions. Indeed, in the absence of the metal ions, a dynamic equilibrium is established 
between the competing processes of association and dissociation of the PAN and QDs, in which only a small fraction of the QDs and PAN molecules are free. In the presence of metal ions, a eompetitive competing process of the formation of PAN/Co or PAN/Ni compounds occurs. When the association constant of this reaction is much greater than that for QD/PAN complexes, the reaction may be considered practically irreversible, leading to a permanent decrease in the number of PAN molecules capable of binding with Zn ions on the surface of the QD shell. Furthermore, we can reasonably assume that the PAN/ion complex formed is unable to bind with to the QDs, since the main metal binding site on the PAN molecule is already occupied by the $\underline{a}$ metal ion. We conclude that the dissociation of a majority of the QD/PAN complexes is expected.

We propose that polymer films with embedded QD/PAN complexes can be used for optical sensing of $\mathrm{Co}^{2+}$ and $\mathrm{Ni}^{2+}$ ions in aqueous solutions. Almost-Practically nonluminescent films with $\mathrm{C}_{\mathrm{QD}}=4 \underline{\mathrm{x}} 10^{-4} \mathrm{M}$ and $\boldsymbol{R}$ of $1: 1$ have been used for the titration experiments. The integrated intensities of the QD PL band were measured after submerging the films for $5 \mathrm{~h}$ in $\mathrm{Co}^{2+}$ and $\mathrm{Ni}^{2+}$ solutions of various concentrations at room temperature. These conditions were chosen to allow completion of the complex dissociation process. Figure 6 demonstrates the ion concentration dependencies of the PL intensity for $\mathrm{Co}^{2+}$ (a) and $\mathrm{Ni}^{2+}$ (b). We found that concentrations of $10^{-8} \mathrm{M} \mathrm{Co}^{2+}$ and $10^{-8} \mathrm{M} \mathrm{Ni}^{2+}$ in water solutions can be determined within experimental errors of $\sim 15 \%$, even without eptimizing optimization of the sensor parameters. These values are at least an order of magnitude lower than the detection limit of 6.7 ngmL $^{-1}$ for Co(II) and 3.2 ngmL $^{-1}$ for Ni(II) obtained by means ofby colorimetric measurements with PAN as a complexing reagent in the aqueous phase using the non-ionic surfactant Tween 80 [15].

The polymer films with QD/PAN complexes do not exhibit any changes in the PL intensity when they are kept in water at $\mathrm{pH} \_$values in the range of $6-13$. This is why the experiments described here were carried out at a $\mathrm{pH}=6.5$. However, we found that the PL from $\theta f$ films appears and grows in intensity with at $\mathrm{pH}$ levels below 5 . This can be explained by the 
PAN $/ \mathrm{Zn}^{2+}$ complex instability in an acid environment [13]. This fact may be exploited when using these polymeric films for optical $\mathrm{pH}$ sensing.

\section{Conclusion}

In conclusion, we have investigated the optical properties of complexes consisting of a luminescent $_{2}$ hydrophobic core/shell CdSe/ZnS quantum dots and 1-(2-pyridilazo)-2-naphtol (PAN) molecules, which are-known as the a colorimetric indicator of Co and Ni ions. Using absorption-luminescence analysis, we showed that, in both in-organic solutions and when embedded in polymer films, the QDs and PAN form a complex where the PAN binds to surface Zn ions in the ZnS QD shells. Due to the strong spectral overlapping between the QDs PL and PAN $/ \mathrm{Zn}^{2+}$ absorption bands, the QD luminescence is quenched via efficient FRET. We found that the presence of $\mathrm{Co}^{2+}$ and $\mathrm{Ni}^{2+}$ ions, both in organic solution or polymeric film, results in the dissociation of the QD/PAN complex due to the formation of stable PAN/Co and PAN/Ni chelates. Significantly, dissociation of QD/PAN complexes is followed by the appearance of QD luminescence, whose intensity increases with the concentration of the metal ions in the water solution $_{2}$ allowing the quantitative photoluminescence sensing of the relevant corresponding metal ions. Incorporation of QD/PAN sensors in hydrophobic polymer films, allowing metal ion diffusion and concentration, together with the utilization of the luminescent response from highly luminescent, hydrophobic QDs, offers considerable promise for the fabrication of highly sensitive $_{2}$ analytical indicators of transition metal ions in aqueous solutions for biological and ecological applications. We suppose-expect that this approach will have application to the that

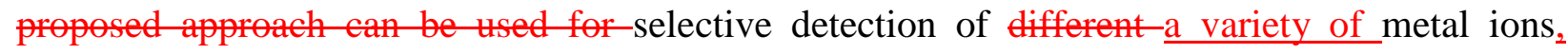
depending on the by a proper selection of QD and molecule-indicator pairs chosen.

\section{Acknowledgments}


This work is supported by the Russian Federal Agency for Education Projects RNP (nos. 2.1.1.1880, 2.1.1.1917 and 2.1.1.1933) and Russian Fund for Basic Research Grants nos. 09-0200333 and 09-02-01439. M.A. was supported by a NATO SfP grant 983207 and the “NANOTECH” program. 


\section{References:}

1. J. M. Costa-Fernandez, Anal. Bioanal. Chem. 384, 37 (2006).

2. J. F. Callan, A. P. De Silva, R. C. Mulrooney and B. Mc Caughan. Journal of Inclusion Phenomena and Macrocyclic Chemistry 58, 257 (2007).

3. K. E. Sapsford, T. Pons, I. L. Medintz, and H. Mattoussi. Sensors, 6, 925-953 (2006).

4. D. F. Boltz, M. G. Mellon, Anal. Chem. 38, 317R (1966).

5. R. Mogul, Luminescent nanosensor, WO patent 2006083269 (2006).

6. D. Betteridge, P. Todd, Q. Fernando, and H. Freiser, Anal. Chem. 35, 729 (1963).

7. N. Alizadeh, A. Moemeni, M. Shamsipur, Anal. Chim. Acta 464, 187 (2002).

8. F. Lazaro, M. D. Luque de Castro, M. Valcarcel, Anal. Chim. Acta 214, 217 (1988).

9. Novacote® Specification, http://www.jolybar.com/SOLVENT\%20BASED.pdf

10. D, V, Talapin, A, L, Rogach, A, Kornowski, M, Haase, H, Weller, NanoLetters 1, 207 (2001).

11. A. Sukhanova, L. Venteo, J. Devy, M. Artemyev, V. Oleinikov, M. Pluot, I. Nabiev, Lab. Invest. 82, 1259 (2002).

12. K. L. Cheng and R. H. Bray, Anal. Chem. 27, 782 (1955).

13. M. Kumar, V. Sharma, L. J. Rao, Food Chem. 52, 7763 (2004).

14. J. R. Lakowicz, Principles of fluorescence spectroscopy, Springer, p. 282 (2006); R. Wargnier, A. V. Baranov, V. G. Maslov, V. Stsiapura, M. Artemyev, M. Pluot, A. Sukhanova and I. Nabiev, Nano Letters 4, 451 (2004); A. O. Orlova, V. G. Maslov, A. V. Baranov, I. Gounko, S. Byrne, Opt. Spectrosc. 105, 726 (2008).

15. G. A. Shar and G. A. Soomro, The Nucleus 41, 77 (2004). 


\section{Figure captions}

Fig. 1. Absorption (1) and PL (2) spectra of CdSe/ZnS QDs in toluene. $\mathrm{C}_{\mathrm{QD}}=5 \underline{\mathrm{X}}=10^{-7} \mathrm{M} \cdot \lambda_{\mathrm{EXC}}=$ $360 \mathrm{~nm}$. (3) - the absorption spectrum of neutral PAN in toluene $\left(\mathrm{C}_{\mathrm{PAN}}=10^{-6} \mathrm{M}\right)$. (4) - the absorption spectrum of a mixture of QDs and PAN in toluene $\left(\mathrm{C}_{\mathrm{QD}}=5 \underline{x} 10^{-7} \mathrm{M}\right.$ and $\mathrm{C}_{\mathrm{PAN}}=10^{-6}$ M) that coincides with the characteristic absorption spectrum of the PAN/ $\mathrm{Zn}^{2+}$ complexes.

Inset: PL spectra of QD and PAN mixture at different $\mathrm{C}_{\mathrm{QD}}$ : $\mathrm{C}_{\mathrm{PAN}}$ ratios $(\boldsymbol{R}): 1$ - 1:0, 2 - 3:1, 3 $2: 1,4-1: 1,5-1: 10 . C_{\mathrm{QD}}=5 \underline{\mathrm{x}} 10^{-7} \mathrm{M} \cdot \lambda_{\mathrm{EXC}}=360 \mathrm{~nm}$.

Fig. 2. PL decay curves of free QD (1) and QD /PAN mixture (2) in toluene at $\boldsymbol{R}$ of 2:1. $\lambda_{\mathrm{EXC}}=$ $405 \mathrm{~nm}, \lambda_{\mathrm{EM}}=530 \mathrm{~nm}$.

Fig. 3. PL (a) and absorption (b) spectra of $2 \mu \mathrm{m}$ thick polymeric films containing free QDs, $\mathrm{QD} / \mathrm{PAN}$ and PAN $/ \mathrm{Zn}^{2+} . \mathrm{C}_{\mathrm{QD}}=4 \underline{\mathrm{x}} 10^{-4} \mathrm{M}$.

(a): 1 - free QDs; 2-5 - QDs/PAN at $\boldsymbol{R}$ of 3:1, 2:1, 1:1 and 1:10, respectively. $\lambda_{\mathrm{EXC}}=360 \mathrm{~nm}$.

(b): 1 - free QDs; 2-3 - QDs/PAN at $\boldsymbol{R}$ of 1:1 and 1:10, respectively; dashed line is the film with $\mathrm{PAN} / \mathrm{Zn}^{2+}$ chelate compound, $\mathrm{C}_{\mathrm{PAN}}=3 \underline{\mathrm{x}} 10^{-3} \mathrm{M}$.

Fig. 4. Absorption spectra of polymeric films with $\mathrm{QD} / \mathrm{PAN}$ complexes $\left(\mathrm{C}_{\mathrm{QD}}=4 \underline{\mathrm{x}} 10^{-4} \mathrm{M}, \boldsymbol{R}\right.$ =1:10): 1 - as prepared; 2 and 3 -after submersion in $5 \underline{x} 10^{-6} \mathrm{M}$ and $10^{-5} \mathrm{M}$ cobalt nitrate solution, respectively. 4 - -the film with PAN after immersion in a cobalt nitrate solution with a concentration of $6 \underline{x} 10^{-6} \mathrm{M}$. The multiplication factors are shown.

Insert: PL spectra of polymeric films with QD/PAN complexes. 1 - as prepared; 2-4 - after submersion in a cobalt nitrate solution with concentrations of $10^{-6} \mathrm{M}, 5 \underline{\times 1} 10^{-6} \mathrm{M}$ and $10^{-5} \mathrm{M}$, respectively. $\lambda_{\mathrm{EXC}}=360 \mathrm{~nm}$. 
Fig. 5. Absorption spectra of polymeric films with $\mathrm{QD} / \mathrm{PAN}$ complexes $\left(\mathrm{C}_{\mathrm{QD}}=4 \underline{\mathrm{x}} 10^{-4} \mathrm{M}, \boldsymbol{R}\right.$ =1:10): 1 - as prepared; 2 and 3 -after submersion in $10^{-5} \mathrm{M}$ and $2 \underline{\mathrm{x}} 10^{-5} \mathrm{M}$ nickel sulfate solutions, respectively. 4__ the film with PAN after submersion in $10^{-5} \mathrm{M}$ nickel sulfate solution. The multiplication factor is shown.

Insert: PL spectra of polymeric films with QD/PAN complexes: 1 - as prepared; 2-4 - after submersion in $10^{-6} \mathrm{M}, 5 \underline{\mathrm{x}} 10^{-6} \mathrm{M}$ and $10^{-5} \mathrm{M}$ nickel sulfate solutions, respectively. $\lambda_{\mathrm{EXC}}=360 \mathrm{~nm}$.

Fig. 6. PL intensity of QD/PAN films $\left(\mathrm{C}_{\mathrm{QD}}=4 \underline{\mathrm{x}} 10^{-4} \mathrm{M}, \boldsymbol{R}=1: 1\right)$ as a function of concentration of (a) $\mathrm{Co}^{2+}$ and (b) $\mathrm{Ni}^{2+}$ in aqueous solutions. Insets show the regions of low ion concentration. $\lambda_{\mathrm{EXC}}=360 \mathrm{~nm}$. 